\title{
In der Schule
}

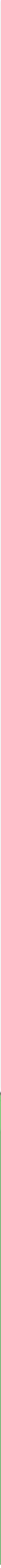

1 Was siehst du? Sprich. 8

2 Höre und sprich nach. (2) (1)

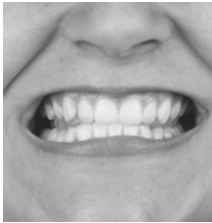

\section{Name}


In der Schule

3a Sprich nach und fahre mit dem Finger nach. (3) () 2
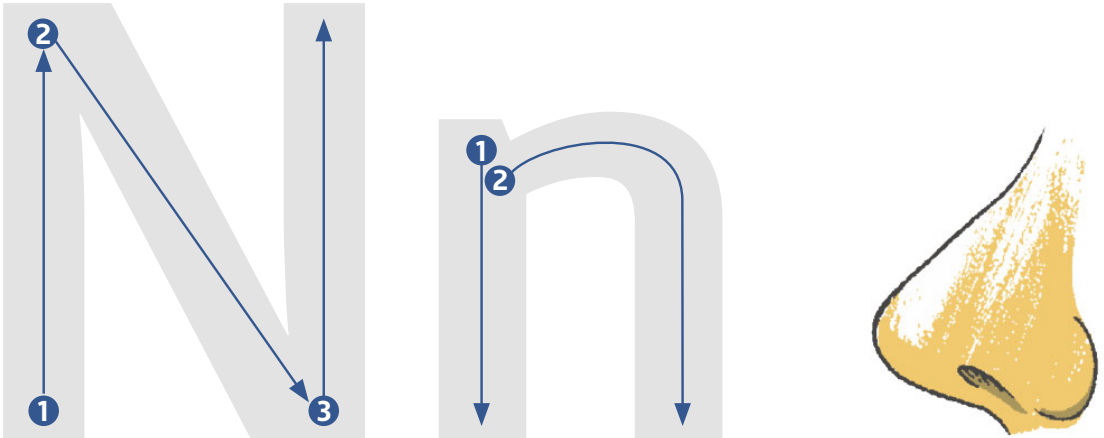

3b Schreibe.

N

n

$\mathrm{Nn}$

Nase

Ananas

Essen

3c Sprich N. Fühlst du die Vibration?

Nnnnnnn. 
4a Sprich nach und klatsche. 8

$\mathrm{Na}$ an sa as da ad An na Sa na $\mathrm{Na}$ sa

$\mathrm{Ne}$ en se es de ed $\mathrm{Ne}$ na $\mathrm{Da}$ na $\mathrm{Na}$ da

4b Zeichne die Silbenbögen ein.

5 In welchem Wort hörst du N n? Kreuze an. (3) (3)
$\mathbf{0} \square$
3
3 $\square$
$\bullet$
o $\square$
• $\square$

6 Wo ist $\mathrm{N}$ n? Höre und schreibe. (3) (4) 4
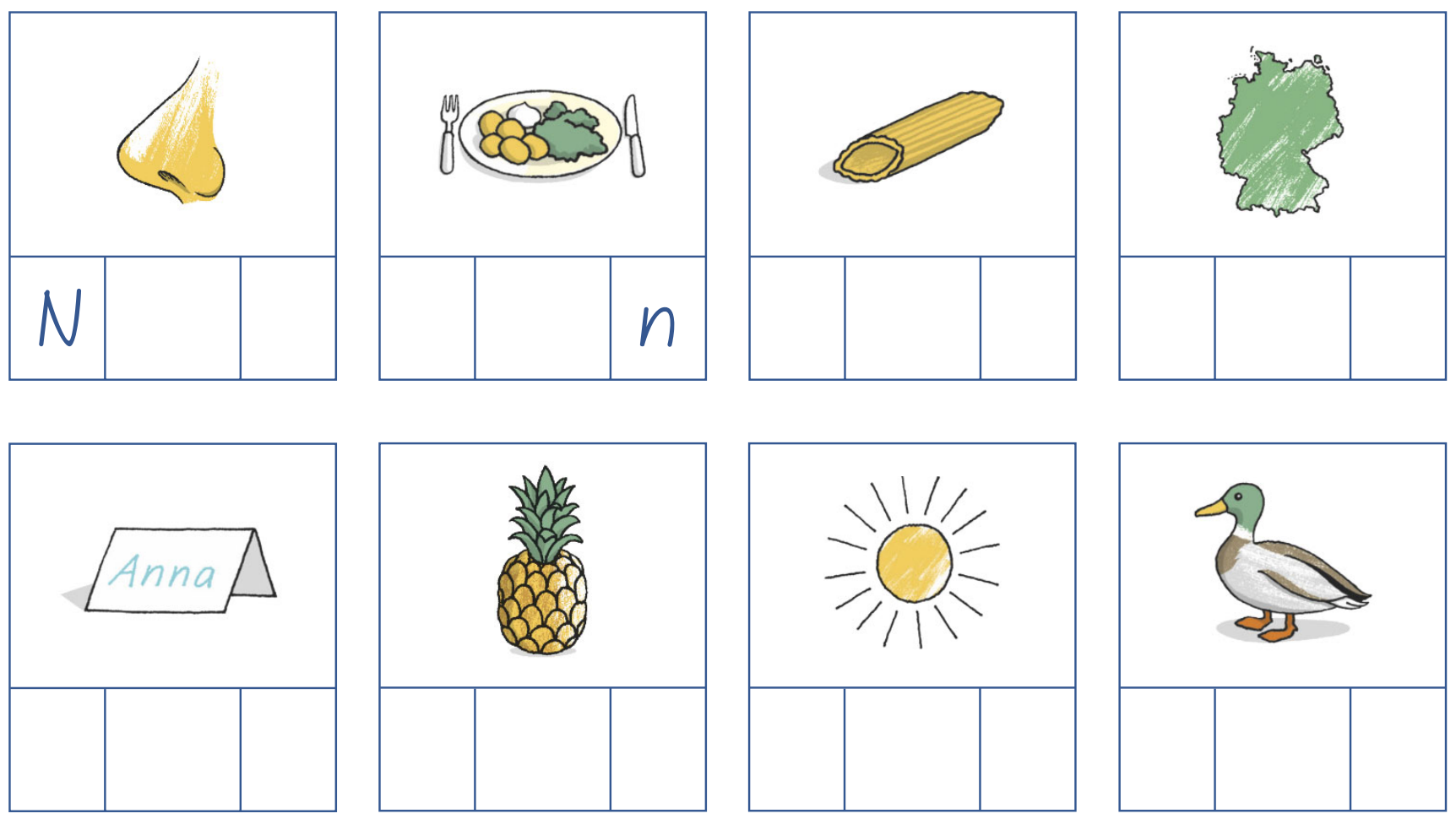


\section{In der Schule}

7a Verbinde.

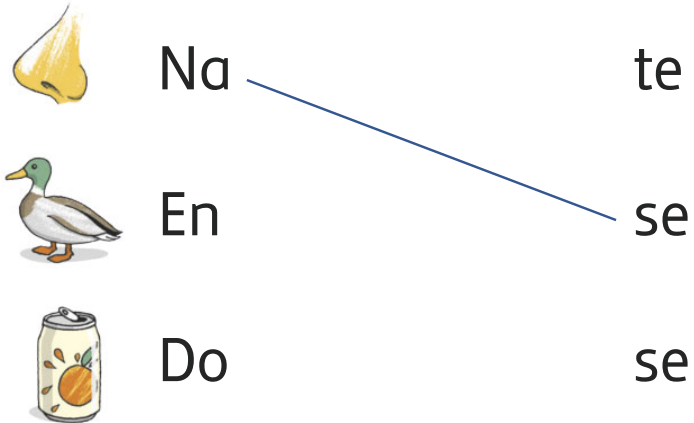

So

ne

Son

del

$\mathrm{Nu}$

$\mathrm{fa}$

7b Höre und schreibe. (2) (\$)

8 Buchstabenecken. Lauft in der Klasse. ( $)$

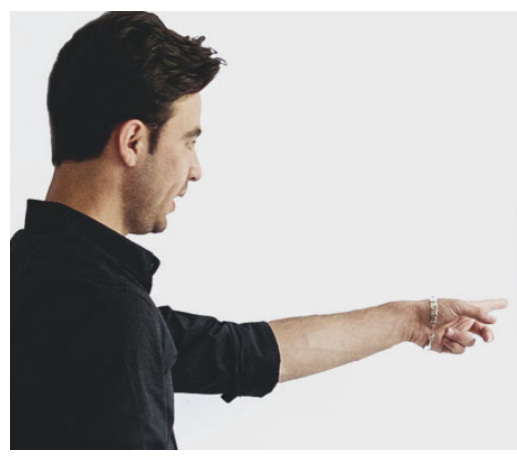

Dd

9 Schreibe $\mathrm{N}$ n.

Gute _ Morge _.

Wie ist Ihr _ame?

Mei __ ame ist Hassa _ Se_i. 
10a Höre und klatsche. (2) (9) 8

10b Schreibt eure Namen in die Liste.

\begin{tabular}{|l|c|}
\hline \multicolumn{1}{|c|}{ Name } & Betonung \\
\hline Francesca & $\bullet \bullet$ \\
\hline Mariam & $\bullet$ \\
\hline Stefan & $\bullet$ \\
\hline & \\
\hline & \\
\hline & \\
\hline & \\
\hline
\end{tabular}

10c Klatscht alle Namen mit der richtigen Betonung.

- laut klatschen - leise klatschen 


\section{In der Schule}

11a Höre und sprich nach. (2) (8)

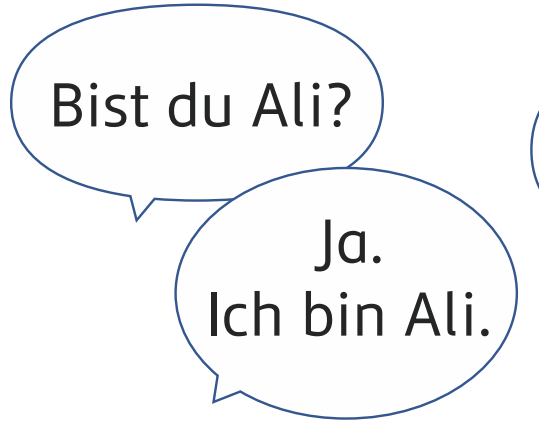

Sind Sie

Frau Seni?

Nein. Ich bin

Elena Danilow.
(1)

du - Vorname

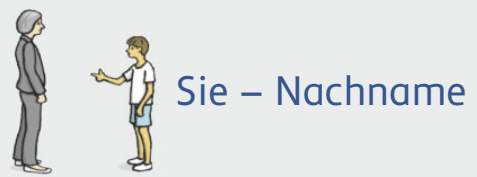

11b Ist der Name richtig? Höre und kreuze an.

\section{(3) (1)}

(Nena Eder

Isa Asisi

Ernst Adam $\square$ ja $\square$ nein

$\square$ ja $\square$ nein

$\square$ ja $\square$ nein

11c Sprecht zusammen.

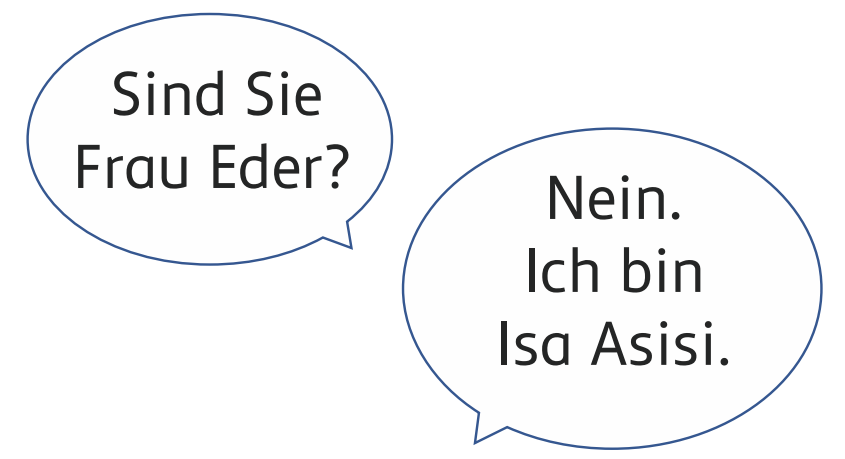


12a Höre und lies mit. (2) (의, 目

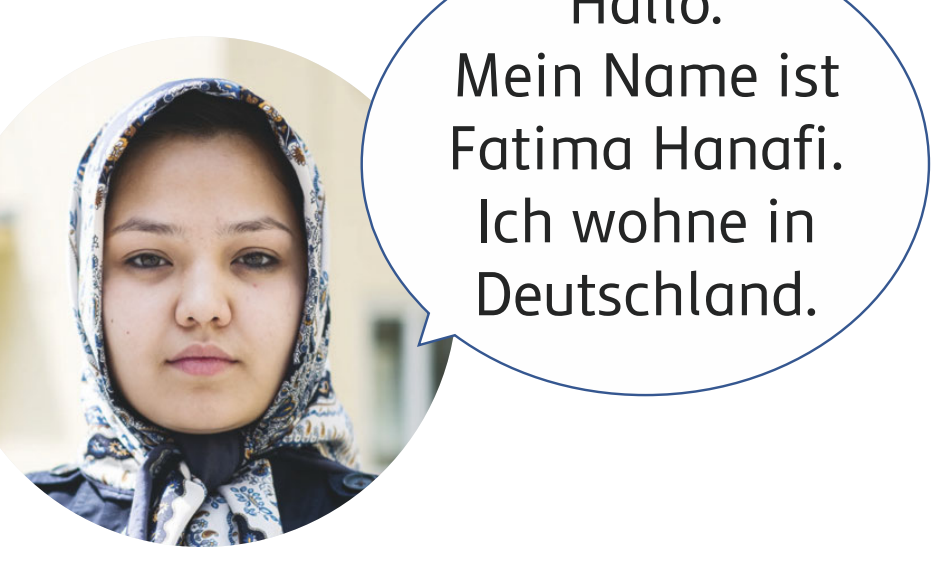

12b Meine Aufgabe für dich. Schreibe das Wort richtig.
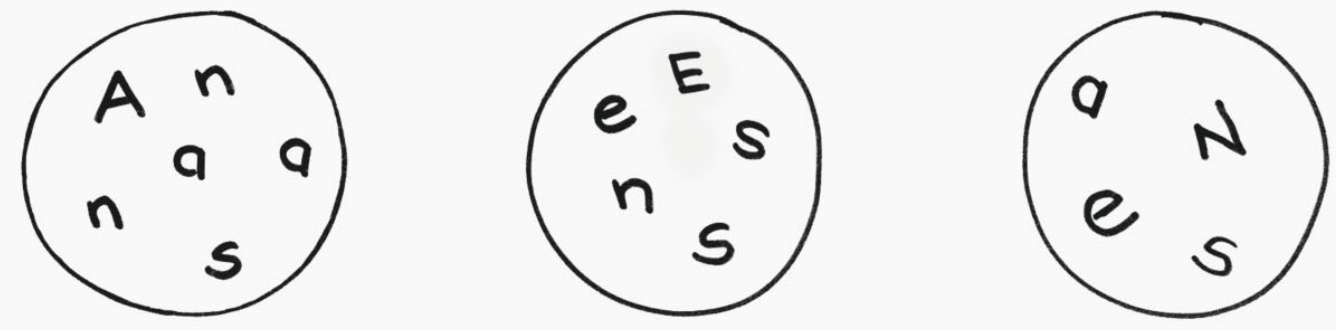

12c Schreibe eine eigene Aufgabe. 
07 Meine Lernseiten

$\star$ Schreibe.

Name

Vorname

Nachname

$\star \star$ Verbinde.

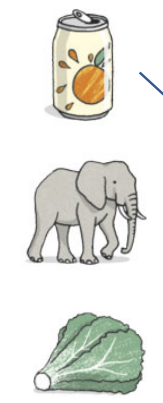

das Sofa

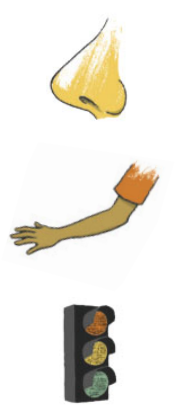

die Ampel

der Salat

die Sonne

die Dose

der Elefant

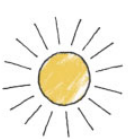

der Arm

die Nase

$\star \star \star$ Schreibe.

Name mit A

Name mit S

Name mit $\mathrm{E}$

Name mit D

Name mit $\mathrm{N}$ 
$\star$ Schreibe.

die Stadt

das Land

der Name

$\star \star$ Verbinde die Silben.

$\begin{array}{llll}\mathrm{Na} & \text { pel } & \text { Sa } & \text { del } \\ \text { So } & \text { se } & \text { Son } & \text { ne } \\ \text { Do } & \text { fa } & \text { Do } & \text { pel } \\ \text { Am } & \text { te } & \text { Am } & \text { se } \\ \text { En } & \text { se } & \text { Nu } & \text { lat }\end{array}$

$\star \star \star$ Schreibe.

der El_fant

der _rm

die _ose

das _ofa

die _ase

$\geqslant 0_{11}=$ die So

e 
固 Mein Wortschatz

Schreibe.

$\sqrt{t a n n a} A$ der Name

die Nase

das Essen

die Nudel

das Land

der Vorname

der Nachname

Lies und sprich.

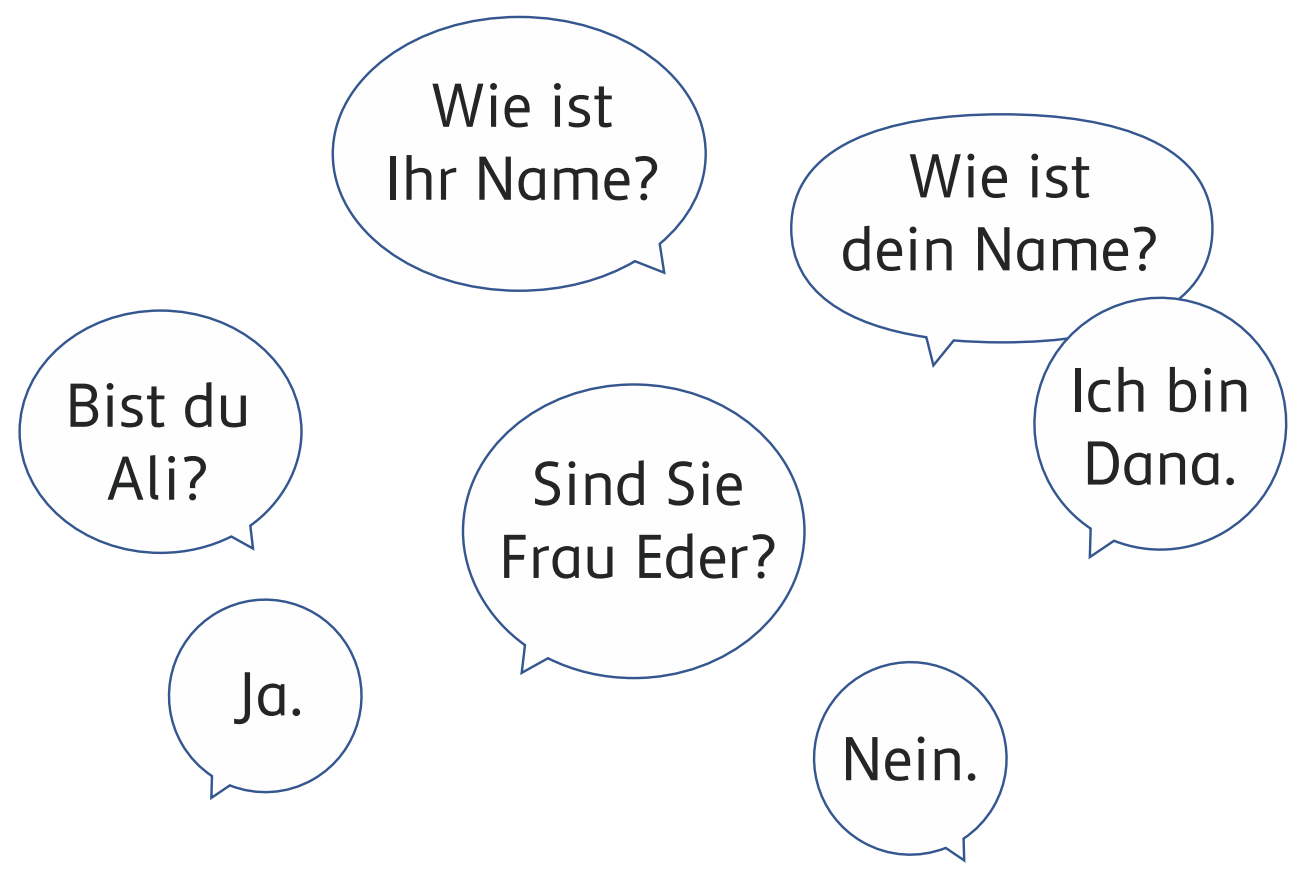




\section{Lernen unterwegs}

Mein Schulausweis.

\section{Schüler/Schülerin}

Vorname

Foto

Nachname

Adresse

Schule

Unterschrift

Datum 
Open Access Dieses Kapitel wird unter der Creative Commons Namensnennung - Nicht kommerziell - Keine Bearbeitung 4.0 International Lizenz (http://creativecommons.org/licenses/by-nc-nd/4.0/deed.de) veröffentlicht, welche die nicht-kommerzielle Nutzung, Vervielfältigung, Verbreitung und Wiedergabe in jeglichem Medium und Format erlaubt, sofern Sie den/die ursprünglichen Autor(en) und die Quelle ordnungsgemäß nennen, einen Link zur Creative Commons Lizenz beifügen und angeben, ob Änderungen vorgenommen wurden. Die Lizenz gibt Ihnen nicht das Recht, bearbeitete oder sonst wie umgestaltete Fassungen dieses Werkes zu verbreiten oder öffentlich wiederzugeben.

Die in diesem Kapitel enthaltenen Bilder und sonstiges Drittmaterial unterliegen ebenfalls der genannten Creative Commons Lizenz, sofern sich aus der Abbildungslegende nichts anderes ergibt. Sofern das betreffende Material nicht unter der genannten Creative Commons Lizenz steht und die betreffende Handlung nicht nach gesetzlichen Vorschriften erlaubt ist, ist auch für die oben aufgeführten nicht-kommerziellen Weiterverwendungen des Materials die Einwilligung des jeweiligen Rechteinhabers einzuholen. 